\title{
Early Power Estimation in Heterogeneous Designs Using SoCLib and SystemC-AMS
}

\author{
François Pêcheux, Khouloud Zine El Abidine, and Alain Greiner \\ UPMC/LIP6/SOC, France \\ francois.pecheux@lip6.fr, \\ khouloud.ZineElAbidine@lip6.fr, \\ alain.greineralip6.fr
}

\begin{abstract}
The presentation will describe a use case that consists in the modeling and simulation of a genuine heterogeneous system composed of individually powered Wireless Sensor Network nodes. The models are written in SoCLib and SystemC-AMS, an open-source C++ extension to the OSCI SystemC Standard dedicated to the description of AMS designs containing digital, analog, RF hardware as well as other disciplines. SoCLib is a library of digital IPs simulation models dedicated to the design of shared memory multiprocessor archutectures. It is currently being extended to support power estimation at the bit-cycle-accurate level of abstraction.

Concretely, a power-aware system of WSN nodes will detailed that can monitor a physical seismic perturbation, transmit information on this perturbation to other nodes by means of $2.4 \mathrm{GHz}$ RF communication links, and finally compute the epicenter of the perturbation by asking the 32-bits processor embedded in a node to solve the system of nonlinear equations relative to the triangulation algorithm. Each node is powered by an autonomous kinetic battery model.
\end{abstract}

